\title{
Marco Assennato, Linee di fuga. Architettura, teoria, politica
}

Palerme, Duepunti, 2011, 152 pages.

\section{Roberta Borghi}

\section{(2) OpenEdition}

\section{Journals}

Édition électronique

URL : http://journals.openedition.org/crau/509

DOI : $10.4000 /$ crau.509

ISSN : 2547-5746

\section{Éditeur}

Éditions du patrimoine

Édition imprimée

Date de publication : 1 septembre 2013

Pagination : 92-94

ISBN : 978-2-7577-0109-6

ISSN : 1296-4077

\section{Référence électronique}

Roberta Borghi, «Marco Assennato, Linee di fuga. Architettura, teoria, politica », Les Cahiers de la recherche architecturale et urbaine [En ligne], 28 | 2013, mis en ligne le 12 septembre 2017, consulté le 24 septembre 2020. URL : http://journals.openedition.org/crau/509 ; DOI : https://doi.org/10.4000/ crau.509 
la construction légère. L'école tisse alors des liens avec un programme tout à la fois concurrent et complémentaire : l'église, qui dès le début du $x x^{e}$ siècle donne également lieu à des constructions d'une extrême simplicité ${ }^{4}$. Sur le plan de la méthode, la place accordée aux acteurs et aux débats peut être lue, elle, comme le discret manifeste d'une autre histoire des types architecturaux, loin du formalisme de Pevsner. En filigrane, enfin, c'est la place accordée à l'enfant dans la société qui est en jeu tout au long de ce récit.

SIMON TEXIER

Historien de l'architecture, professeur d'histoire de l'art contemporain, université de Picardie Jules Verne.

\section{Marco Assennato, Linee di fuga. Architettura, teoria, politica,}

Palerme, Duepunti 2011 152 pages.

\section{Lignes de fuite.} Architecture, théorie, politique Philosophe et chercheur en sciences politiques et en architecture, Marco Assennato articule son texte autour d'une position forte : face à la complexité de la nature humaine et de ses manières d'habiter le monde contemporain, le croisement de l'architecture, de la théorie et de la politique pourrait ouvrir de nouvelles perspectives comme des lignes de fuite sur la conception et la construction de notre futur.

Cela correspondrait à un acte nécessaire de refondation de la société et de ses espaces habités, qui prendrait la mesure autant des paradigmes de la modernité (évoqués à la fois par nostalgie ou par opposition) que de l'impasse théorique dans laquelle nous vivons aujourd'hui.

La structure du livre est celle d'une pièce en trois actes, avec un prologue, qui présente le champ de réflexion, et un épilogue qui ouvre des pistes concrètes de travail.

En ouverture, l'architecte et historienne Françoise Very met l'accent sur la valeur du futur proposée par l'auteur qu'il voit comme une capacité de penser et de construire la diversité et la complexité plutôt qu'une expression d'un progrès linéaire et idéologique. Le courage de " prendre le risque de penser le monde en tant que futur " critique les "bonnes pensées de l'écologie et de l'environnement ", devenues aujourd'hui des phénomènes de mode, qui risquent de masquer - via une négation de la ville ou via une surévaluation des techniques - la nécessité de redéfinir, sur des bases théoriques autres, l'architecture et la politique. Dans le prologue, Assennato analyse les notions sur lesquelles sa théorie s'appuie, en se positionnant par rapport au débat culturel actuel. Ce travail remarquable sur les mots et leurs étymologies permet de prendre la mesure de la richesse des contenus, ainsi que des éléments encore insuffisamment éclaircis, afin d'élaborer une théorie nouvelle, anti-idéologique. L'Architecte est donc celui qui connaît le principe (arché) de la technique (techné), et qui dirige ceux qui I'appliquent (ouvriers). Cela constitue une première hiérarchie politique entre l'architecte, qui connaît la cause des choses (la théorie) et I'ouvrier, qui l'applique (pratique) ${ }^{1}$. L'outil qui permet de traduire les principes théoriques en des réalisations concrètes est le Projet, relu ici dans sa double origine : le Proiectus latin exprime l'idée d'une action intentionnelle, qui est à la fois projective et prédictive. L'Ent-wurf allemand exprime un cadre théorique différent, où l'origine est mise en question ${ }^{2}$. Selon les deux acceptions, le projet est donc un travail de renouvellement constant de
4. On renverra à ce propos aux travaux de Pierre Lebrun, notamment à son ouvrage, Le temps des églises mobiles. L'architecture religieuse des Trente Glorieuses, Gollion, Infolio, 2011
1. Jacques Derrida, Point de folie Maintenant l'architecture, dans Bernard Tschumi, La case vide : La Villette 1985, Londres, Architectural Association, 1986.

2. Massimo Cacciari, "Progetto", Laboratorio politico $n^{\circ} 2$, Turin, mars-avril 1981, p. 88-119. 
nos fondements, de transformation de l'existant et, en même temps, de prévision du futur. L'Architecture est ainsi la discipline qui, dans ce processus, opère une synthèse entre théorie et pratique, principes et techniques, mémoire et oubli, espace et temps (kairòs). La Politique est bien plus que la " gestion des intérêts d'une communauté ${ }^{3}$ "; face aux crises actuelles, elle devient plutôt la «forme non-consensuelle de l'agir collectif, dans laquelle le désaccord produit l'entrée en scène des subjectivités »(Jacques Rancière). La politique est donc le synonyme du pouvoir démocratique, alors que la police s'oppose à elle via la cristallisation, la limitation et la gestion de ce pouvoir. L'architecture, avec sa double nature technico-théorique, devrait se situer au centre du débat politique, en en partageant les faits, les ouvertures, les conflits. D'où le lien entre les deux disciplines.

Pourtant, les rôles de l'architecture et de la politique semblent aujourd'hui de plus en plus marginaux et impuissants vis-à-vis d'autres pouvoirs, tels que l'économie ou la finance.

Si nous considérons la culture moderne comme la dernière qui a su donner à l'architecture et à la politique ce pouvoir, nous comprenons aussi pourquoi, dans les trois actes centraux du livre, Marco Assennato cherche à analyser les raisons de l'état de crise actuel, à la lumière de différentes tentatives de dépassement de la modernité.

3. Jacques Rancière, Aux bords du politique, Paris, Gallimard, 1998, p. 10.
D'abord, " une polémique sous l'angle de la nostalgie », regarde la crise de l'architecture contemporaine à travers les positions des architectes italiens Franco La Cecla et Vittorio Gregotti. Face à I'« inutilité » de I'architecture actuelle (coincée entre les contradictions de la modernité et la superficialité du Star system), La Cecla ${ }^{4}$ propose de sortir définitivement de la discipline architecturale et de s'ouvrir à une nouvelle approche interdisciplinaire, fondée sur l'« habitat et l'essence narrative des espaces ». Marco Assennato y voit une attitude descriptive et passive par rapport au projet, qui révèle la nostalgie de la valeur de l'architecture ancienne. Gregotti ${ }^{5}$ cherche à défendre l'autonomie de l'architecture, en proposant la redéfinition d'une théorie du projet, fondée sur la construction et I'habitabilité. La refondation disciplinaire de Gregotti révèle une nostalgie de la modernité, en cherchant à réinventer et à accomplir ses idéologies inachevées. D'après Assennato, les deux formes de nostalgie étant liées à une image du monde qui ne correspond plus à celle complètement modifiée et artificielle dans laquelle nous vivons aujourd'hui, elles ne peuvent pas représenter la réponse pour le futur.

Ensuite, «Modernité et conflit», critique la lecture stéréotypée de la modernité faite par le Postmoderne, qui ne considèrerait pas, d'après l'auteur, son autocritique et sa remise en question. À partir d'une analyse des figures les plus représenta-

4. Franco La Cecla, Contre l'architecture, Paris, Arléa, 2011 (éd. italienne Contro I'architettura, Turin, Bollati Boringhieri, 2008). tives de la modernité, l'auteur évoque la pluralité des thèmes débattus : la restructuration de la société due à l'émergence des masses; les tentatives de contrôle de cette force sociale via les thèmes de I'habitat, du logement et de sa gestion, à l'échelle individuelle, de la ville et du territoire; les liens entre la technique et la société ; la présence de certaines " contre-mesures conceptuelles » comme l'idée que la technique est nécessaire mais non suffisante ou comme la distinction entre l'utile et le rationnel ; la définition d'une méthode fondée sur l'équilibre entre limites différentes, le beau et l'utile, le social et le privé, l'art et la technique (Walter Gropius) ; la relation entre l'esthétique et l'éthique dans I'essentialité du détail constructif (Mies van der Rohe) ou dans la relation entre architecture, nature et être humain (Frank Lloyd Wright). Marco Assennato retrouve en Manfredo Tafuri, une réponse à la faillite des Modernes ${ }^{6}$. L'« opérativité de la critique " nous permettra de nous insérer et de répondre au conflit permanent qui caractérise la contemporanéité.

Enfin, "Lignes de fuite du postmoderne » analyse les différentes trajectoires de la culture postmoderne. II y est évoqué sa double nature radicale et progressiste, réactionnaire et opprimante, intéressée par la différence, la complexité tout en étant attachée aux logiques du Marché et de la consommation. Dans la revue Casabella, un débat intense s'impose entre 1982 et 1985 : Massimo Cacciari, Gianni

5. Vittorio Gregotti, Contro la fine dell'architettura, Turin, Einaudi, 2008.

6 Manfredo Tafuri, Projet et utopie: de l'avant-garde à la métropole, Paris, Dunod, 1979 (éd. italienne Progetto e Utopia. Architettura e sviluppo capitalistico, Rome-Bari, Laterza, 1973). 
Vattimo et François Lyotard discutent sur la valeur des pensées "fortes » et des pensées "faibles», en tant que réponses à la crise des grands récits de la modernité. Jacques Derrida et Peter Eisenman proposent une "résistance marginale ", où l'hédonisme individuel et l'expérimentation personnelle prennent la place du conflit historique. Jean Baudrillard et Jean Nouvel évoquent une " théorie hyperréaliste ", où la singularité, I'instant et l'urbanisation virtuelle façonnent la vie contemporaine. Rem Koolhaas élabore sa théorie de la Bigness et du Junkspace, qui sont les expressions des modes de vie de l'espace métropolitain indéfini.

Dans l'épilogue, le livre trace de nouvelles lignes de fuite pour une théorie de l'architecture. Pour sortir du paradigme moderne, la notion de "commun " - ce qui n'est pas privé, ni public - est proposée comme une des trajectoires possibles. En effet, l'origine du terme, le latin "munus », accueille l'idée du don à offrir aux autres, ce qui implique une réciprocité et une mutualité nouvelles. Contrairement aux logiques individualistes de la modernité, le " commun » renvoie à une richesse commune, autant matérielle (l'eau, la nature) que immatérielle (la société, les connaissances, les langages). Cela permettrait aussi d'établir des expressions de l'agir collectif (politique) et d'édifier des formes d'habitat (architecture / ville / territoire), véritablement ouvertes à l'espace et au temps du futur.
Ainsi, conçu par un « non-architecte », le livre a le mérite de proposer une relecture du rôle de l'architecture contemporaine, en travaillant sur une actualisation critique de ses termes et en confirmant sa responsabilité civile face à l'histoire et à la société. II reste peut-être une incertitude par rapport aux outils et aux formes concrètes d'action (aux architectes et aux politiciens de les trouver ?) et aux limites spatio-temporelles suggérées par l'auteur. Comme le théoricien Franco Berardi l'évoque dans la conclusion du livre, le cadre de travail de Marco Assennato est encore une fois la ville occidentale, ce qui pourrait limiter l'élan vers le futur soutenu par le texte. Une ouverture intéressante pourrait alors s'appuyer sur d'autres contextes spatiaux et sociaux, ainsi que sur d'autres croisements entre architecture, théorie et politique.

ROBERTA BORGHI

Architecte-urbaniste, enseignante à l'Énsa de Strasbourg et à l'université de Parme, membre de I'UMR Architecture, morphologie/morphogenèse urbaine et projet, Énsa et Insa de Strasbourg, et du laboratoire Architecture, musées, réseaux, université de Parme, département d'Ingénierie civile et architecture.

\section{Paolo Amaldi, Architecture, profondeur, mouvement}

Gollion, Infolio, coll. «Projet et Théorie », 2012, 495 pages.

L'ouvrage de Paolo Amaldi, Architecture Profondeur Mouvement, deuxième de la collection "Projet et Théorie », représente une somme non pas historique - bien qu'il emprunte le cours de l'histoire architecturale en Occident depuis le Quattrocento italien de Brunelleschi et Alberti jusqu'à Giedion et Le Corbusier mais, nous dirons, "épistémologique ». En effet, ce n'est pas tant l'enchaînement des "faits " comme événements stylistiques (œuvres remarquables, courants, périodes) qui importe que le fonds conceptuel sous-jacent à leur avènement. Ce n'est pas non plus un ouvrage qui emprunterait à une psychologie de la perception (comme chez Arnheim et ses références à la Gestalttheorie) une démarche cognitive appliquée à ces mêmes faits architecturaux, bien que par son titre et son point de départ on pourrait le supposer (par exemple, les références à Descartes, à Locke ou Berkeley, à Merleau-Ponty présent dès la préface, etc.). En fait, ceux-ci sont l'amorce et les points de référence d'une problématique caractérisée comme analyse épistémologique proprement architecturale de l'espace où ces notions (historiques, psychologiques) sont pour nous l'enjeu. 